\title{
Corrosion Evaluation Using Clustering Method Based on Eddy Current Pulsed Thermography
}

\author{
Peizhen SHI a, Song DING ${ }^{\text {a, }}$, Yuming CHEN ${ }^{\text {a }}$, Yiqing WANG ${ }^{\text {a }}$, Guiyun TIAN ${ }^{\text {b }}$, \\ Fangjun $\mathrm{ZHU}^{\mathrm{a}}$ \\ a School of Electrical Engineering and Control Science, Nanjing Tech University, \\ Nanjing, Jiangsu, 211816, China \\ ${ }^{\mathrm{b}}$ School of Engineering, Newcastle University, Newcastle upon Tyne, NE1 7RU, UK
}

\begin{abstract}
As a popular defect of steel, corrosion had been a big challenge to industry safe and structural health. For atmosphere corrosion characterization and evaluation, a clustering by fast search and find of density peaks (CFSFDP) algorithm, combined with gap statistic (GS) method is utilized to corroded Q235 carbon steel tubes. With the proposed method, three natural atmosphere corroded samples are investigated and classified. The proposed method successfully identifies the samples with different service periods. The temperature gradient, which indicates the heat generation and conductivity, is used to analyze cluster center selection. The matching rate is presented as a feature to reflect the corrosion state difference.
\end{abstract}

Keywords. Eddy current pulsed thermography, corrosion evaluation, clustering method.

\section{Introduction}

As the most widely used material, metal has been applied to social and industrial infrastructures. But corrosion induced fatigue and failures cause fatal disasters which cost billions of dollars per year in the U.S. alone [1], and $40 \%$ mechanical failure due to corrosion in the world. To ensure safety, corrosion evaluation which helps re-protecting the material, avoiding accidents and saving lives and money, is expected. To understand corrosion behavior, many chemical and physical methods, such as electrochemical impedance spectroscopy (EIS), scanning Kelvin probe (SKP), scanning acoustic microscopy (SAM) and scanning electrochemical microscopy (SECM), were employed [2]. On the other hand, corrosion state monitoring is more meaningful for in-service equipment and structures. Therefore, many nondestructive testing and evaluation (NDT\&E) technologies were applied, such as ultrasonic testing (UT), eddy-current testing (ECT)[3], radiographic testing (RT) and electromagnetic methods[1-8]. Compared to other technologies, eddy current pulsed thermography (ECPT), a novel NDT\&E technology that provides an opportunity for efficient nondestructive scanning, has been applied to defect detection and characterization for conductive material [9-11].

\footnotetext{
${ }^{1}$ Corresponding author, School of Electrical Engineering and Control Science, Nanjing Tech University, Nanjing, Jiangsu, 211816, China; E-mail: dingsong@njtech.edu.cn
} 
Aijun Yin et al.[12] interpreted the time responses of ECPT image sequences for two physical processes, accumulation of Joule heating and heat diffusion, from general behavior of ECPT and developed feature extraction and pattern recognition for defect characterization. Yunze He et al.[2] employed ECPT to detect blister and ruptured blister areas. Signal processing algorithms, such as PCA, independent component analysis (ICA) and fast Fourier transform (FFT), are applied for feature extraction to distinguish the ruptured blister. Phase thermography analysis method was proposed by Ruizhen Yang et al. [13] for in-situ early marine corrosion detection, sizing, and monitoring. However, the complex corrosion process and oxide layers [14] bring out fuzzy effects on material properties, such as resistance, electrical conductivity, thermal conductivity, permeability and surface emissivity, which makes it difficult to evaluate the corrosion state based on the ECPT technology.

Previous work of the ECPT technology mostly focused on the transient response and lost sight of the relationship between pixels, which indicates the metallic properties, such as thermal conductivity, electrical conductivity, and permeability. Hence, the general objective of this letter is to characterize the corrosion state by clustering the temperature gradient distribution without any model training.

\section{Experimental System and methodology}

\subsection{Corrosion Samples}

For industry enterprises, Q235 carbon steel is widely used to carry dry gas under atmospheric pressure. In this study, three Q235 carbon steel tubes with different atmosphere corrosion, which have exposed to the damp atmosphere for 3 (sample 1), 10 (sample 2) and 14 months (sample 3) as shown in Fig. 1., are selected for investigation. All of these samples have the same diameter of $50 \mathrm{~mm}$. The wall thickness of sample 1 is $3 \mathrm{~mm}$ and the other two are less than $3 \mathrm{~mm}$ due to corrosion.

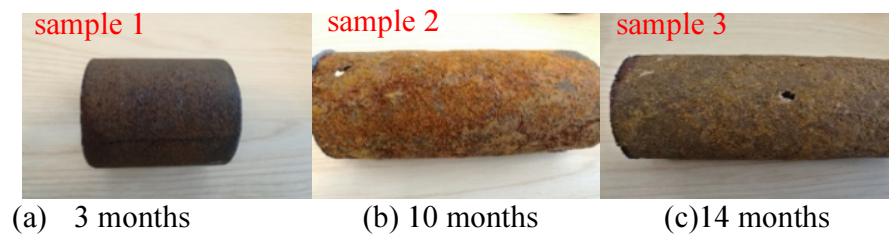

(a) 3 months

(b) 10 months

(c) 14 months

Figure 1. Corrosion samples of Q235 steel with different service periods

Obviously, sample 1 has different apparent conditions to sample 2 and sample 3. But for surface visual inspection, it is hard to estimate the corrosion state of sample 2 and sample 3. In this study, all three samples are measured by ECPT system, and the IR images sequence is analyzed to evaluate the corrosion states which are compared with service period of the samples.

\subsection{Experiment System}

The diagram of ECPT system is shown in Fig. 2. A function generator provides synchronizing pulse which starts the infrared (IR) camera when the induction heater driving the coil. The eddy current supplier, induction heater, drives excitation coil which 
generates high-frequency eddy current on the surface of conductive samples. Then, Joule heat is generated by the inductive eddy current and resistance of the material. An infrared camera is employed for capturing the thermal radiation from the samples surface. In order to understand the thermal distribution and heat conductivity process in the sample, infrared image sequence is collected and stored by special software for further analysis.

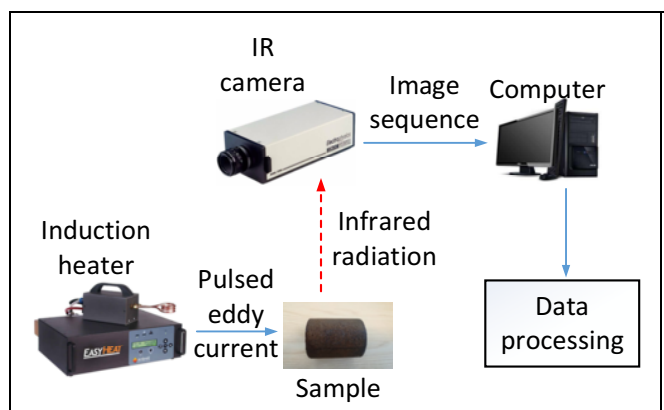

Figure 2. ECPT system diagram

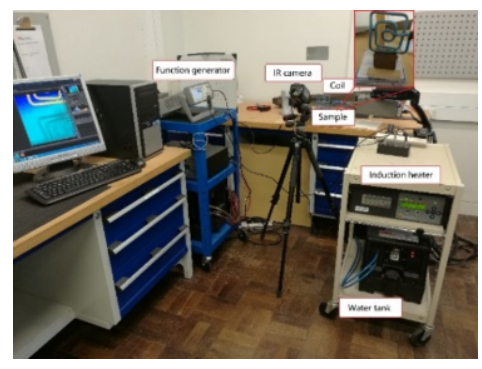

Figure 3. ECPT experiment system

Fig. 3 shows the experimental system for corrosion samples testing which was performed at Newcastle University. The eddy current supplier, EasyHeat 224 from Cheltenham Induction Heating, provides $380 \mathrm{~A}_{\mathrm{rms}}$ alternative excitation current (the maximum current is $400 \mathrm{~A}_{\mathrm{rms}}$ ), and excitation frequency from $150 \mathrm{kHz}$ to $400 \mathrm{kHz}$. In order to limit the inductive eddy current on samples' surface, which contributes to a shallow heat resource, $260 \mathrm{kHz}$ excitation frequency is selected based on skin depth effect. The rectangular excitation coil is made of $6.35 \mathrm{~mm}$ high-conductivity hollow copper tube which connected to a pump for water-cooling. FLIR SC655, an uncooled infrared camera with a $640 \times 120$ array of $7.5 \mu \mathrm{m}$ InSb detector, is applied to acquire the IR image sequence. IR images are acquired with a $200 \mathrm{~Hz}$ sampling rate and $30 \mathrm{mK}$ temperature sensitivity. For synchronizing the excitation current and IR camera acquisition, a square pulse is generated by a function generator, Keysight $33621 \mathrm{~A}$, to start EasyHeat 224 and FLIR SC655.

\subsection{Framework}

For understanding the characterization of the corrosion state, Fig. 4 proposes an overall framework for the study process. It can be divided into three parts. The first part is data acquisition. As shown in Fig.4 part 1, ECPT technology is applied for obtaining thermal images sequence.

Because the raw thermal image data contains large information and some redundant data, it is not suitable for data analysis directly [15]. Generally, feature extraction can be performed on the original thermal images for reducing data and facilitating subsequent analysis. Thus, the second part is feature extraction, which contains area selection, data normalization, polynomial fitting and principal component analysis (PCA). The reasons why the four feature extraction methods are down for the raw thermal images are as follows. During thermal image analyzing, it is necessary to take into account the nonuniform heating of the ECPT technology. At the same time, the area far from the coil is not suitable for analysis because of weak eddy current and low Joule heat generation. The area around the coil was used for analysis due to the heat generation and dissipation 


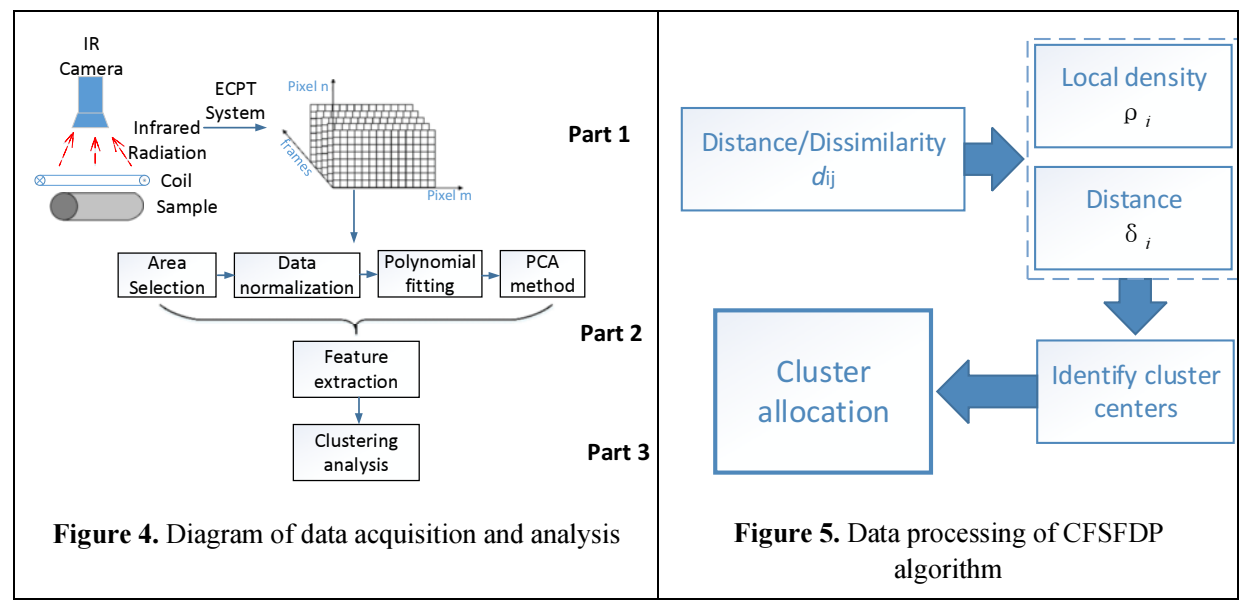

reflect the material properties. After the area selection, data normalization can observably reduce the lift-off and bias light effect and is helpful to compare the trend of temperature increase and decrease. Since the data acquired from the ECPT system is always discrete and along with noise, the polynomial fitting method can effectively overcome these two problems. Meanwhile, the parameters of the polynomial fitting function can be used to represent the discrete temperature points, and data are greatly compressed. After the polynomial fitting, there is still a lot of redundant information. Therefore, PCA method is applied to reduce the data dimensions. After that, the temperature sequence are normalized between 0 and 1. Finally, the clustering by fast search and find of density peaks is employed for data analysis.

\subsection{Clustering by fast search and find of density peaks algorithm}

Clustering is the basic composition in data analysis pipelines. Among these algorithms, Clustering by fast search and find of density peaks (CFSFDP) [16] is a novel densitybased clustering algorithm that can detect non-spherical data sets with few parameters. The algorithm is based on two assumptions about the cluster centers, which must both have a higher density than their neighbors and a relatively large distance from points with higher local density [16].

In this study, CFSFDP is utilized to analyze the global information of the material surface temperature with different corrosion stage, as shown in Fig.5. For every point on the surface of the material, two parameters need to be calculated by the dissimilarity/distance $d_{i j}$ between data point $i$ and point $j$. The calculation of the local density of $\rho_{i}$ of point $i$ is given by equation 1 .

$$
\rho_{i}=\sum_{j=1}^{m} \chi\left(d_{i j}-d_{c}\right)
$$

Where $\left\{\begin{array}{ll}\chi(x)=1 & x<0 \\ \chi(x)=0 & x \geq 0\end{array}, d_{c}\right.$ is a cutoff distance. The distance $\delta_{i}$ of point $i$ is defined as:

$$
\delta_{i}=\min _{j: \rho_{j}>\rho_{i}} d_{i j}
$$


By convention, for the point with the highest density, it is defined as $\delta_{i}=$ $\max _{j}\left(d_{i j}\right)$.

\section{Result and discussion}

As clarified above, the IR images are analyzed by Matlab 2010 software. One typical IR image is shown in Fig. 6, which is the infrared distribution at $200 \mathrm{~ms}$ after excitation eddy current application.

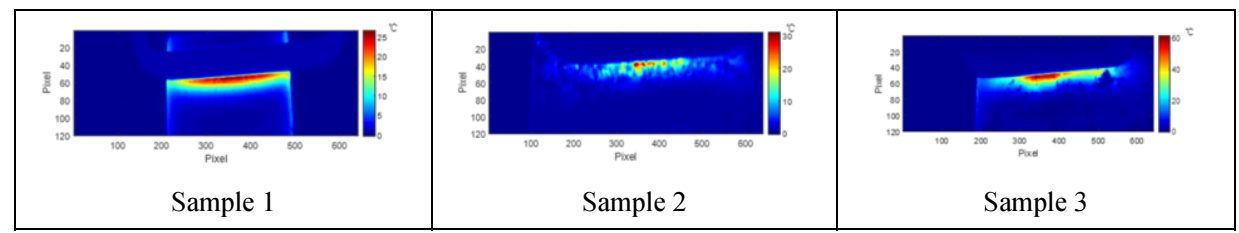

Figure $6.40^{\text {th }}$ frame of IR image sequence for three samples

Firstly, all three samples are clustered using the CFSFDP algorithm. As shown in Fig. 7(a), obviously, there are three points that are more outstanding, which have larger $\delta$ and $\rho$. Thus, these three points are selected as cluster centers. Fig. 7(e) shows the clustering results. Referring to Fig. 7, the clustering algorithm can basically identify the areas in the data sets.

To verify the clustering algorithm, the clustering results are compared with the actual distribution. As shown in Table 1, the matching rate, correct clustering percentage, of the algorithm is $85.24 \%$, which represents the confidence of the CFSFDP algorithm for characterizing the three corrosion samples. For each sample, 1452 points are selected from the infrared image while only 53 points of sample 1 and 140 points of sample 3 are in the error cluster, respectively. On the contrary, sample 2 shows the lowest matching rate due to 457 error clustered points.

Table 1. The matching matrix of clustering with actual distribution for stage 1, stage 2 and stage 3

\begin{tabular}{ccccc}
\hline & Cluster 1 & Cluster 2 & Cluster 3 & Total matching rate \\
\hline Stage 1 & 1399 & 0 & 53 & \\
Stage 2 & 10 & 995 & 447 & $85.24 \%$ \\
Stage 3 & 30 & 110 & 1312 & \\
\hline
\end{tabular}

To further explore the effectiveness of the algorithm for only two corrosion states, different two samples are selected, clustered and compared. Fig. 7 shows the decision graphs and corresponding clustering results. As shown in this figure, the cluster centers can be easily chosen, and the corresponding clustering results divide data points into two categories for each clustering. In the same way, the matching matrix of clustering groups for different two samples are calculated for comparison. 


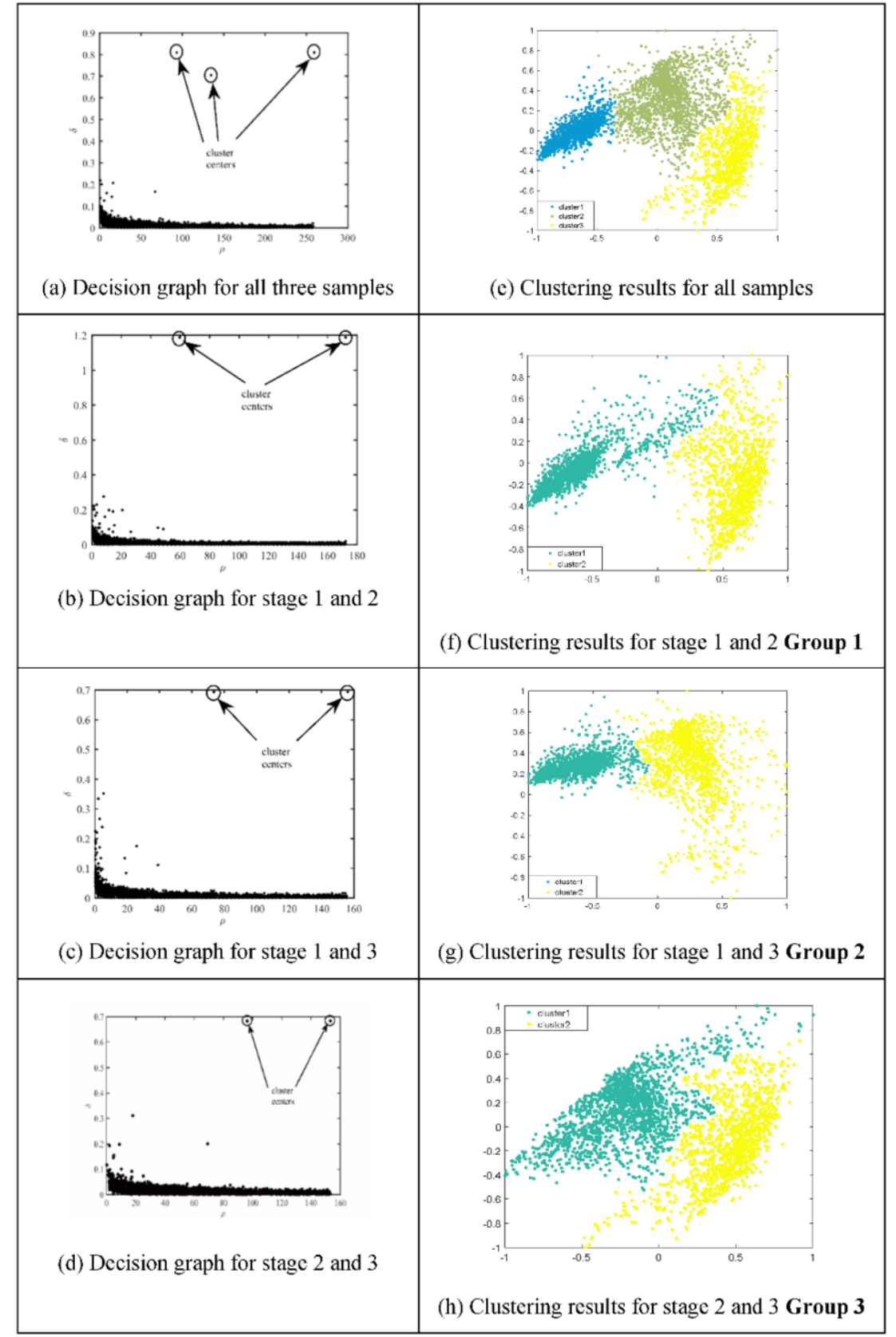

Figure 7. Decision graphs and Clustering results for different clustering groups

From fig. 7 (b) (d), the distance between cluster centers of clustering 3 significantly smaller than that of group 1 and 2. Accordingly, group 3 has the lowest matching rate, $81.71 \%$. Longer service means bigger variation in the mechanical properties, such as conductivity and emissivity, which makes the feature points distributed more discretely. 
As shown in figs. 7 (f) (h), sample 1 provides obviously concentrated distribution, while sample 2 distributes as discrete as sample 3 due to their similar service periods.

\section{Conclusion and future work}

As corrosion affects the material properties, this paper investigates the CFSFDP clustering method based on ECPT technology for inspecting Q235 steel tubes' corrosion state. Temperature gradient distribution is applied for cluster centers selection. After that, CFSFDP algorithm is used to recognize the corrosion state based on the PCA features. All the points in the inductive region are treated indistinguishably and without any training model. Furthermore, matching rates are calculated for different clustering groups, which is proposed as a new feature to characterize the corrosion state after different service periods.

CFSFDP algorithm shows its contributions to corrosion state characterization and evaluation. Moreover, the results show this approach is more credible for two corrosion state recognition than three or more samples distinction. Thus, detailed researches for understanding and quantitative analyzing are expected to improve this clustering approach in future work.

\section{Acknowledgment}

The authors wish to express their sincere gratitude to Prof. Tian, Dr. Kongjing Li for contributing to experiment guide and case study. This work was supported by the Jiangsu Province Natural Science Foundation for the Youth (No. BK20180687).

\section{References}

[1] Fangxin Zou, Frederic B. Cegla. On quantitative corrosion rate monitoring with ultrasound. Journal of Electroanalytical Chemistry. 2018; 812: 115-121.

[2] Yunze H, Guiyun Tian and Mengchun Pan et al. An investigation into eddy current pulsed thermography for detection for corrosion blister. Corrosion Science. 2014; 78: 1-6.

[3] Yunze He, Guiyun Tian and Hong Zhang et al. Steel Corrosion Characterization Using Pulsed Eddy Current Systems. IEEE Sensors Journal. 2012; 12(6): 2113-2120.

[4] M. Yasri, B. Lescop and E. Diler et al. Fundamental basis of electromagnetic wave propagation in a Zinc microstrip lines during its corrosion. Sensors and Actuators B: Chemical. 2016; 223: 352-258.

[5] Keiji Tsukada, Yuta Haga and Koji Morita et al. Detection of Inner Corrosion of Steel Construction Using Magnetic Resistance Sensor and Magnetic Spectroscopy Analysis. IEEE Transaction on Magnetics. 2016; 52(7), 6201504.

[6] Jenifer Alcántara, Daniel de la Fuente and Belén Chico et al. Marine Atmospheric Corrosion of Carbon Steel: A Review. Materials. 2017; 10, 406.

[7] Ivan S. Cole. Recent Progress and Required Developments in Atmospheric Corrosion of Galvanised Steel and Zinc. Materials. 2017; 10, 1288.

[8] Hong Zhang, Leng Liao and Jianting Zhou et al. The Non-Destructive Test of Steel Corrosion in Reinforced Concrete Bridges Using a Micro-Magnetic Sensor. Sensors. 2016; 16, 1439.

[9] Yunze He, Mengchun Pan and Guiyun Tian et al. Eddy current pulsed phase thermography for subsurface defect quantitatively evaluation. Appl. Phys. Lett. 2013; 103,144108.

[10] Kongjing Li, Guiyun Tian and Liang Cheng et al. State Detection of Bond Wires in IGBT Modules Using Eddy Current Pulsed Thermography. IEEE Trans. on Power Electronics. 2014; 29(9): 5000-5009.

[11] Libing Bai, Bin Gao and Guiyun Tian et al. Spatial and Time Patterns Extraction of Eddy Current Pulsed Thermography Using Blind Source Separation. IEEE Sensors Journal. 2013; 13 (6): 2094-2101. 
[12] Aijun Yin, Bin Gao and Guiyun Tian et al. Physical interpretation and separation of eddy current pulsed thermography. Journal of Applied Physics. 2013; 113, 064101.

[13] Ruizhen Yang et al. Through coating imaging and nondestructive visualization evaluation of early marine corrosion using electromagnetic induction thermography. Ocean Engineering. 2018; 147: 277-288.

[14] U.S. Department of Energy. DOE Fundamentals Handbook, Chemistry Module 2 Corrosion. Washington, D. C. 1993. 140p.

[15] Bin Gao, Xiaoqing Li and W.L. Woo et al. Quantitative validation of Eddy current stimulated thermal features on surface crack. NDT \& E International. 2017; 85: 1-12.

[16] A. Rodriguez and A. Laio, Clustering by fast search and find of density peaks. Science. 2014; 334(6191): 1492-1496. 